\title{
Left Partial Anomalous Pulmonary Venous Return in an Isymptomatic Adult
}

\author{
Tarun Jain ${ }^{1,2}$, Shahzaib Nabi', Jainil Shah', Javier Sanchez ${ }^{1}$, Sagger Mawri ${ }^{1}$ \\ 'Henry Ford Hospital Health System, Detroit, Michigan; ${ }^{2}$ Heart and Vascular Institute, \\ Loyola University Medical Center, Maywood, Illinois.
}

\begin{abstract}
:
Partial anomalous pulmonary venous return (PAPVR) encompasses a specific group of rare congenital cardiovascular anomalies that are caused by the abnormal return of one or more, but not all, of the pulmonary veins directly to the right atrium or indirectly through a variety of venous connections from the anomalous pulmonary vein. It is frequently associated with other congenital heart disease, most commonly atrial septal defect. The overall incidence is estimated to be between $0.4 \%-0.7 \%$. In patients with isolated multiple pulmonary vein involvement, signs and symptoms are dependent upon the degree of left-to-right shunting. Hereby, we highlight the utility of cardiac magnetic resonance imaging as a reliable non-invasive tool in diagnosis of rare congenital anomalies along with planning the management.
\end{abstract}

Key words: Congenital Heart Defects, Heart Septal Defects, Pulmonary Veins, Magnetic Resonance Imaging.

A cardiac magnetic resonance scan was performed on a 32-year old asymptomatic male referred for further evaluation of cardiac murmur. The study demonstrated normal left ventricular size and function along with a mildly dilated right ventricle and atrium. No atrial septal defect was present. Pulmonary vein angiography demonstrated normal right sided pulmonary venous anatomy along with two pulmonary veins receiving blood from the left inferior lobe of the lung. The left superior pulmonary vein was seen draining into the left innominate vein [Fig. 1,2]. The findings on the pulmonary vein angiography established the diagnosis of left partial anomalous pulmonary venous return (PAPVR). On phase contrast images, pulmonary to systemic flow ratio (Qp:Qs) was 1.5, consistent with a hemodynamically significant left to right shunt.
PAPVR refers to the abnormal return of one or more, but not all of the pulmonary veins draining directly or indirectly into the right atrium through a variety of venous connections from the anomalous pulmonary vein [1]. The prevalence of PAPVR is between $0.4 \%-0.7 \%$ with left sided PAPVR occurring in 10\%-18.2\% of all reported PAPVR cases [2]. The hemodynamic burden is believed to be minimal when only one vein connects anomalously explaining the frequent incidental discovery of the anomaly in asymptomatic patients as in our patient [3].

Cardiac magnetic resonance imaging, in addition to acquiring high resolution images of the pulmonary and systemic veins, allows for a comprehensive and quantitative evaluation of right

\section{Corresponding Author: Dr. Shahzaib Nabi}

Email: snabi1@hfhs.org

Received: April 15, 2015 | Accepted: June 23, 2015 | Published Online: June 30, 2015

This is an Open Access article distributed under the terms of the Creative Commons Attribution License (creativecommons.org/licenses/by/3.0)

Conflict of interest: None declared | Source of funding: Nil | DOI: http://dx.doi.org/10.17659/01.2015.0071 

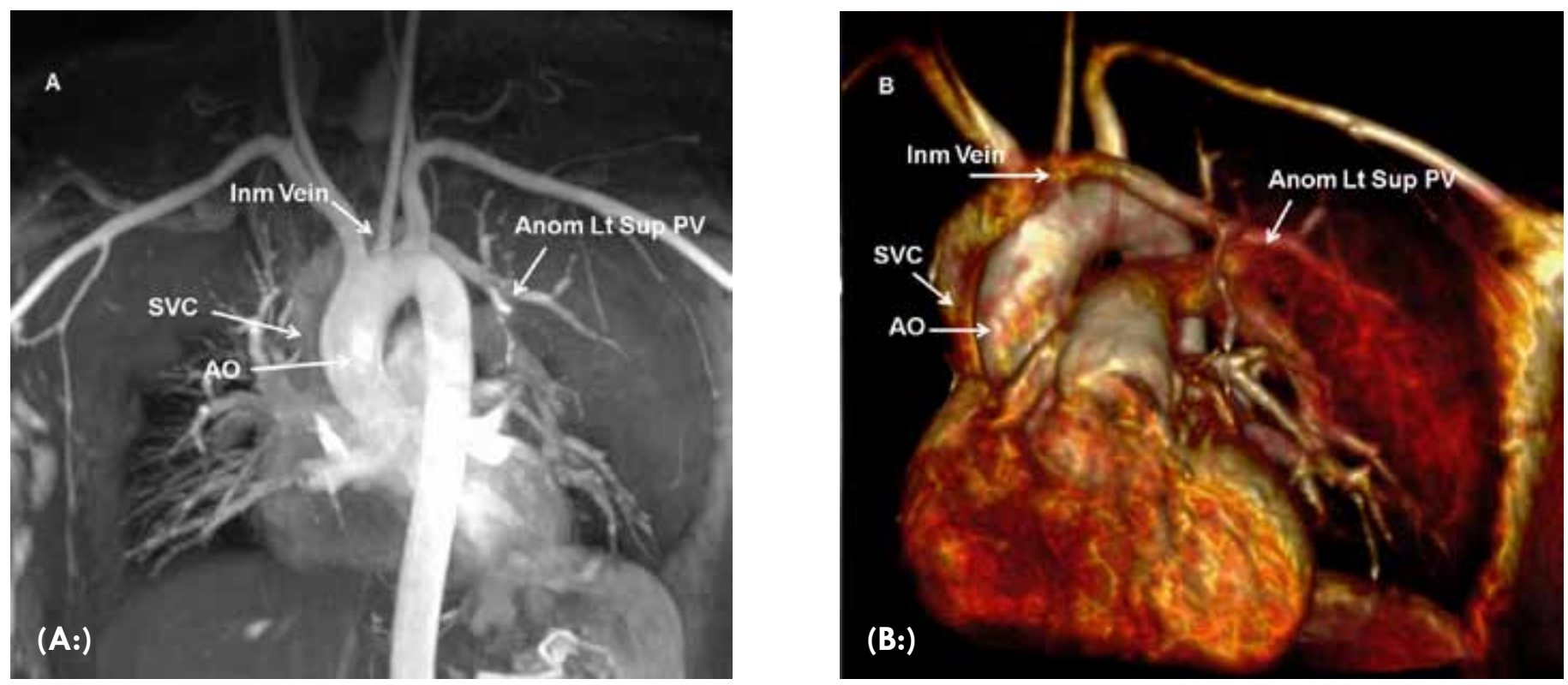

(A:) Pulmonary vein MRA, (B:) 3D volume rendered MRA. These images demonstrate left superior pulmonary vein draining into the left innominate vein consistent with the diagnosis of left partial anomalous pulmonary venous return. MRA: magnetic resonance angiography; AO: aorta; Inm vein: innominate vein; SVC: superior vena cava; Anom Lt Sup PV: anomalous left superior pulmonary vein.

ventricular volume and function. Moreover, CMR helps in the quantification of intra-cardiac shunts by calculating the Qp:Qs ratio [3]. Thus, CMR proves to be a robust non-invasive tool in the evaluation of patients with vascular anomalies and is also helpful in planning the management.

\section{References}

1. Kivisto S, Hanninen $H$, Holmstrom M. Partial anomalous pulmonary venous return and atrial septal defect in adult patients detected with 128-slice multidetector computed tomography.
Journal of Cardiothoracic Surgery. $2011 ; 6: 126$.

2. Ammash NM, Seward JB, Warnes CA, Connolly HM, O'Leary PW, Daniel GK. Partial anomalous pulmonary venous connection: diagnosis by transesophageal echocardiography. Journal of American College of Cardiology. 1997; 29:13518.

3. Dyme JL, Prakash A, Printz BF, Kaur A, Parness IA, Nielsen JC. Physiology of Isolated Anomalous Pulmonary Venous Connection of a Single Pulmonary Vein as Determined by Cardiac Magnetic Resonance Imaging. Am Journal of Cardiology. 2006; 98:107-110. 